\title{
Evaluación, diseño y propuestas de sistemas de ventilación en la rehabilitación de edificios residenciales españoles. Estudio de caso
}

\section{Evaluation, design and proposals for ventilation systems in refurbishment of Spanish residential buildings: Case study}

$\underline{\text { A. Meiss }}^{(*)}$, J. Feijó-Muñoz ${ }^{(*)}$, M. A. Padilla-Marcos ${ }^{(*)}$

\section{RESUMEN}

En los últimos años, la rehabilitación de edificios ha experimentado un gran impulso, haciendo hincapié en aspectos constructivos y energéticos, pero omitiendo los relativos a la ventilación. Esto se debe a la dificultad de conocer la ventilación natural disponible y en las escasas soluciones derivadas del DB-HS3.

Este trabajo pretende exponer una metodología para, a partir de simulaciones numéricas y datos climáticos, obtener el gradiente estacional de presiones en los cerramientos y, conocida su permeabilidad mediante ensayos de presurización in situ, obtener los caudales disponibles en tres períodos del año (verano, invierno y media anual).

Para el cumplimiento de la exigencia HS3 es recomendable acudir al carácter prestacional del CTE, que admite otras soluciones alternativas al Documento Básico, pero es necesario introducir una serie de cambios para la solución propuesta de proyectar un sistema individual de ventilación con caudal variable o Ventilación Cruzada Controlada.

Palabras clave: Ventilación; rehabilitación de edificios; calidad del aire; ensayo de presurización.

\section{ABSTRACT}

In recent years, building retrofitting has grown in importance, due to the need to improve construction and energy conditions, but omitting those relating to ventilation. This is due to the difficulty to estimate the available natural ventilation and the limited solutions derived from $\mathrm{DB}-\mathrm{HS}_{3}$.

This work aims to present a methodology, through numeric simulations and climate data, able to solve the seasonal pressure gradient in the building' envelope and, once known the airtightness with on-site pressurization tests, the calculation of the ventilation flows in three representative conditions (summer, winter an annual average).

To fulfill the HS3 requirement is advisable to use the performance-based nature of CTE, which allows other alternative solutions to the DB. But it is necessary to introduce changes in the regulations to allow the proposed solution of an individual ventilation system with variable flow or Controlled Cross Ventilation.

Keywords: Ventilation; building retrofit; indoor air quality; pressurization test.

${ }^{(*)}$ G.I.R. Arquitectura \& Energía. E.T.S. Arquitectura. Universidad de Valladolid. Persona de contacto/Corresponding author: meiss@arq.uva.es (A. Meiss)

Cómo citar este artículo/Citation: Meiss, A., Feijó-Muñoz, J., Padilla-Marcos, M. A. (2016). Evaluación, diseño y propuestas de sistemas de ventilación en la rehabilitación de edificios residenciales españoles. Estudio de caso. Informes de la Construcción, 68(542): e148, doi: http://dx.doi.org/10.3989/ic.15.101.

Licencia / License: Salvo indicación contraria, todos los contenidos de la edición electrónica de Informes de la Construcción se distribuyen bajo una licencia de uso y distribución Creative Commons Attribution License (CC BY) Spain 3.o. 


\section{INTRODUCCIÓN}

De manera general, la rehabilitación de edificios ha experimentado un importante impulso por la toma de conciencia del patrimonio existente en los núcleos urbanos. Así, las Administraciones Públicas han fomentado esta labor mediante actuaciones de incentivo económico tendentes a mejorar las condiciones energéticas y de confort en viviendas construidas bajo otros condicionantes menos exigentes.

Simultáneamente los investigadores han caracterizado el parque residencial de la postguerra y el desarrollismo para optimizar los criterios de intervención. De manera general, se ha ahondado en los aspectos constructivos y energéticos de los cerramientos, y en la eficiencia y seguridad de los sistemas (1) (2). El aspecto energético ha sido predominante incluso al abordar el caso de la ventilación natural e infiltraciones, antes que en garantizar la calidad del aire interior.

La calidad del aire, que es un parámetro fundamental del confort humano, se venía confiando tradicionalmente a la apertura voluntaria de las ventanas y a la elevada permeabilidad de carpinterías y cerramientos. Sin embargo, la evolución de los hábitos de uso de los usuarios, caracterizados por largos períodos de ausencia durante el día, ha provocado que estos procedimientos de ventilación sean cada vez menos efectivos. Así, en viviendas monitorizadas, ventiladas de forma manual, se ha comprobado que la calidad del aire empeora notablemente en invierno, aumentando los niveles de concentración de $\mathrm{CO}_{2}$ y de humedad relativa (3). Además, la aleatoriedad de los factores climáticos provoca una renovación no controlada a evitar.

Desde la entrada en vigor del CTE, España dispone de una normativa prestacional que cuantifica con carácter general los parámetros que garantizan esa calidad. El problema surge cuando se quieren aplicar a la rehabilitación unos procedimientos inicialmente dirigidos a la obra nueva, que nada tienen que ver con los supuestos del proyecto original. La consecuencia es que el proyectista recurre en exceso al criterio de flexibilidad, recogido en la Ley 8/2013 de rehabilitación, regeneración y renovación urbanas, amparándose en que la aplicación del CTE no es técnica o económicamente viable o que es incompatible con la naturaleza de la intervención.

El principal problema al que se enfrenta el proyectista es el de cualificar y cuantificar los caudales disponibles de ventilación natural, para la correcta repercusión en los procesos de control energéticos y de calidad del aire. Su desconocimiento, ante la ausencia de métodos simplificados de cálculo fiables, desalienta su toma en consideración, lo que lleva a utilizar de manera habitual sistemas mecánicos, basados en ventiladores que extraen el aire interior de manera continuada. En consecuencia, conocer los caudales disponibles de ventilación natural permite entender la interacción del edificio con el entorno, la influencia de parámetros singulares (altura relativa de la vivienda, número de conductos verticales, permeabilidad de carpinterías), así como proponer alternativas de diseño compatibles con la exigencia normativa de calidad del aire interior.

Este trabajo recoge dicho análisis aplicado a un caso de estudio del proyecto europeo CITyFiED (RepliCable and InnovaTive Future Efficient Districts and cities), pudiendo servir como metodología para afrontar intervenciones en la rehabilitación de edificios atendiendo al parámetro de la calidad del aire interior.

\section{CASO DE ESTUDIO}

El proyecto CITyFIED, cofinanciado por el VII Programa Marco de la UE, tiene como propósito desarrollar e implementar tecnologías y metodologías innovadoras para la rehabilitación de edificios. El proyecto incluye la renovación integral de tres distritos urbanos en Laguna de Duero-Valladolid (España), Soma (Turquía) y Lund (Suecia). La intervención en Laguna de Duero consiste en la rehabilitación de la Urbanización Torrelago (Figura 1), que consta de 31 edificios (1.488 viviendas) construidos entre 1977 y 1981. Cada bloque tiene 12 pisos $(\mathrm{B}+12)$, con 4 viviendas por planta, y una superficie total de intervención de $140.000 \mathrm{~m}^{2}$ a rehabilitar entre 2014-2017.

Existen tres tipos de edificios en forma de $\mathrm{H}$, denominados A, B y C, con una distribución de viviendas que responden a dos tipos. Una más pequeña, de tres dormitorios, estancia, cocina y dos baños, toda con huecos abiertos a fachada. Otra más grande, de cuatro dormitorios, estancia, cocina y dos baños, toda con huecos abiertos a fachada, salvo uno de los baños que es interior (Figura 2). El Bloque A es de cuatro viviendas pequeñas por planta, el Bloque $\mathrm{C}$ de cuatro viviendas grandes por planta y el Bloque B tiene por planta dos pequeñas y dos grandes. A efectos de ventilación, todas las habitaciones tienen carpinterías practicables al exterior, y ciertos locales húmedos (cocinas y aseos interiores) disponen de conducto colectivo tipo shunt, que evacúa en cubierta (algunos de ellos obstruidos o anulados por los propietarios).

Evidentemente estos edificios datan de una fecha muy anterior a la promulgación del CTE y, en consecuencia, no tienen
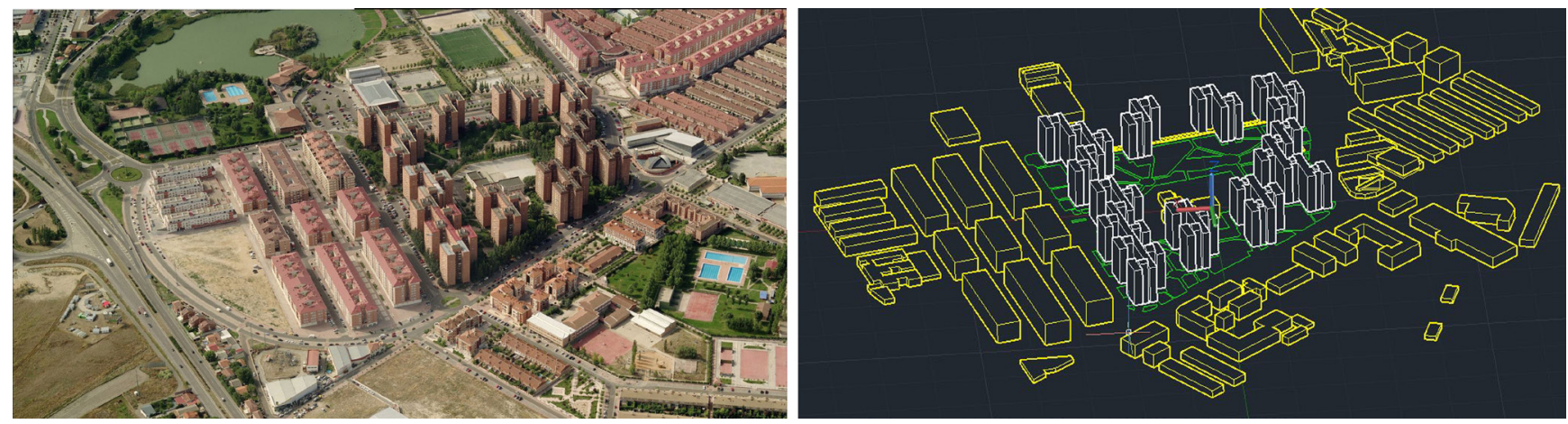

Figura 1. Vista aérea del entorno de Torrelago y ámbito de la simulación. 

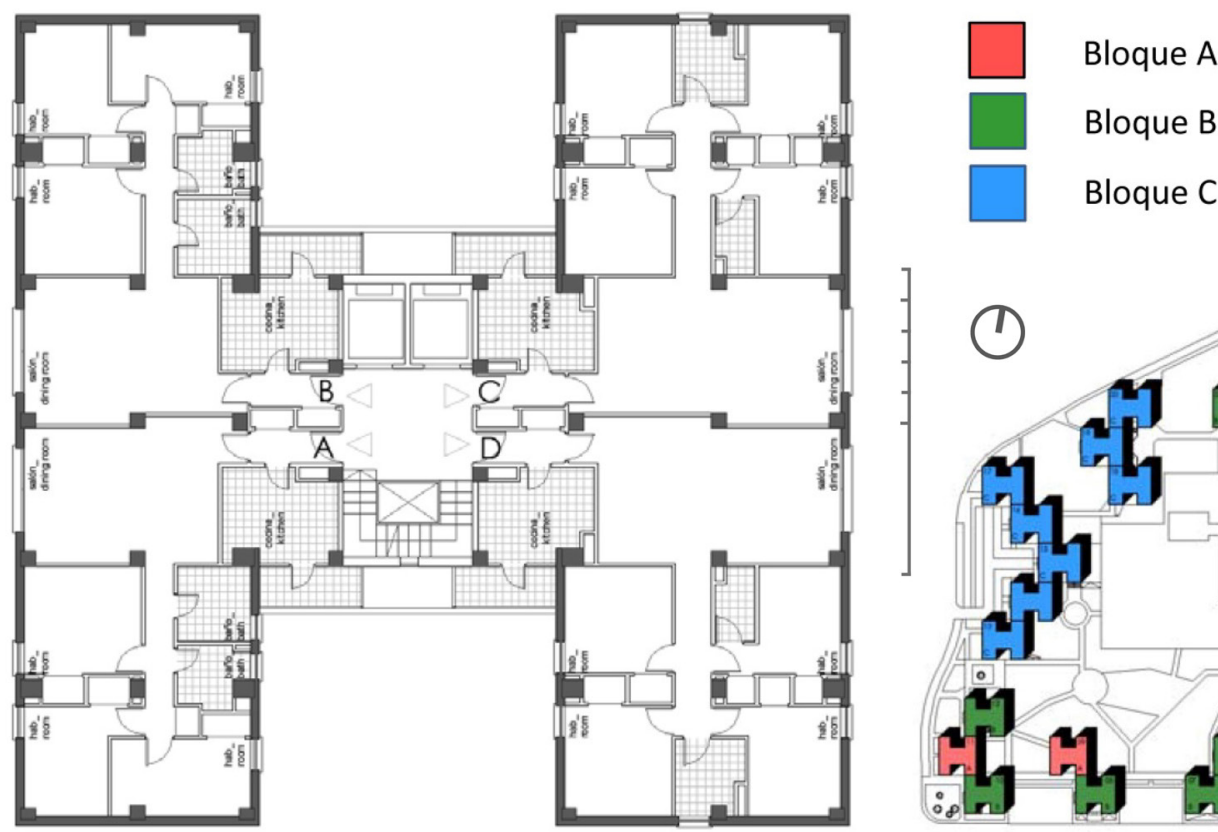

Figura 2. Bloque Tipo B con viviendas de 3 y 4 dormitorios - Tipología en Urbanización.

en proyecto ningún sistema de ventilación. En su rehabilitación habría que remitirse a la exigencia básica HS3 de calidad del aire interior.

\section{METODOLOGÍA DE CÁlCULO DE LA VENTILACIÓN NATURAL}

Los modelos empíricos habitualmente utilizados (4) (5) (6) para predecir la tasa de ventilación no son apropiados para estudios de casos de edificios en altura. En su lugar hay que recurrir a modelos macroscópicos donde sea posible combinar ecuaciones de masa y energía, relaciones empíricas y semiempíricas relativas a la disipación, condiciones de contorno y condiciones zonales in situ, para obtener un sistema de ecuaciones representativo del movimiento del aire en el interior del edificio. Aplicar este modelo a nivel de bloque aislado nos permite evaluar el nivel de máxima disponibilidad de ventilación natural en las viviendas, mientras que el estudio a nivel de distrito cuantifica la variación de dichos caudales en cada bloque del conjunto respecto a considerarlo como entidad aislada.

\subsection{Caracterización del modelo y del emplazamiento}

A pesar que no es posible definir una estrategia de aplicación genérica, se pueden enunciar una serie de principios a considerar. Primero, habrá que caracterizar adecuadamente el entorno y cómo éste influye en las condiciones de la ventilación natural. La entrada de aire exterior al interior del edificio se fundamenta en un diferencial de presiones, que es el resultado del efecto combinado de dos procesos simultáneos: una convección natural por diferencial de densidades por efecto de las distintas temperaturas a ambos lados del cerramiento, y una convección forzada, de origen natural por viento (o artificial en sistemas mecánicos).

La inspección de los bloques de Torrelago nos permite verificar que las condiciones iniciales del proyecto se han visto modificadas por sus propietarios a lo largo de los años, lo que obliga a una nueva caracterización. Entre ellas está el determinar la hermeticidad de la envolvente arquitectónica mediante ensayos de presurización en viviendas representativas.

Las condiciones ambientales adoptadas y sus valores son los específicos del emplazamiento de la urbanización, que perfeccionan los valores estándar de referencia (Tabla 1). Respecto al viento, el dominante a nivel regional es el denominado Ábrego, de componente WSW. Para obtener el perfil específico del viento se recurre al atlas eólico nacional (8), donde se representa la rosa de los vientos a una altura de $80 \mathrm{~m}$. (Figura 3). La velocidad media estacional a dicha altura es de $5,30 \mathrm{~m} / \mathrm{s}$ en invierno, $4,55 \mathrm{~m} / \mathrm{s}$ en verano y $5,02 \mathrm{~m} / \mathrm{s}$ anual. Las direcciones predominantes WSW/SW y NE inciden en los bloques de manera sensiblemente ortogonal.

La urbanización se encuentra en un área de interfaz entre las zonas suburbana y urbana, pudiéndose identificar cuatro entornos:

- Tipo 1: área urbana estructurada al este de la urbanización, con bloques residenciales de 5 y 6 alturas, separados por corredores urbanos.

- Tipo 2: entorno urbano de baja densidad y alturas, y en combinación con áreas verdes.

- Tipo 3: área de densidad media con alternancia de bloques de 2 y 3 plantas y viviendas unifamiliares adosadas.

- Tipo 4: entorno rural, con limitada presencia de masas arbóreas y la laguna que da nombre al municipio.

\subsection{Presiones térmicas}

Por regla general, cuando se verifica una diferencia de temperaturas, ésta genera un cambio en las densidades del aire, que se traduce en un gradiente de presión que origina el tránsito de aire a través de los cerramientos. Tal fenómeno, que asume particular importancia en los edificios altos, se denomina efecto chimenea. A una altura determinada, digamos $Z_{n}$, el gradiente $\Delta p$ es igual a cero, y esta altura se denomina línea 
Tabla 1. Condiciones ambientales de Laguna de Duero (Valladolid).

\begin{tabular}{|l|l|}
\hline EMPLAZAMIENTO & $\begin{array}{l}\text { Laguna de Duero } \\
\left(41^{\circ} 344^{\prime} 59^{\prime} \mathrm{N} \text { y } 704 \mathrm{msnm}\right)\end{array}$ \\
\hline Gravedad de cálculo & $\mathrm{g}=9,80094 \mathrm{~m} / \mathrm{s} 2$ \\
\hline presión atmosférica de referencia & $\mathrm{p}_{\mathrm{a}}=93049 \mathrm{~Pa}$ \\
\hline
\end{tabular}

\begin{tabular}{|c|c|c|c|}
\hline \multicolumn{4}{|c|}{ Condiciones exteriores medias (7) } \\
\hline & Invierno (enero) & Verano (julio) & Anual \\
\hline Temperatura media & $\mathrm{TM}=3, \mathrm{O}^{\circ} \mathrm{C}$ & $\mathrm{TM}=20,5^{\circ} \mathrm{C}$ & $\mathrm{TMA}=11,4^{\circ} \mathrm{C}$ \\
\hline HR media & $\mathrm{HR}=85,3 \%$ & $\mathrm{HR}=46,9 \%$ & $\mathrm{HR}=65 \%$ \\
\hline $\begin{array}{l}\text { Velocidad media } \\
\text { estacional de referencia }\end{array}$ & $5,30 \mathrm{~m} / \mathrm{s}$ & $4,55 \mathrm{~m} / \mathrm{s}$ & $5,02 \mathrm{~m} / \mathrm{s}$ \\
\hline \multicolumn{4}{|c|}{ Condiciones interiores de confort en viviendas } \\
\hline Temperatura & \multicolumn{3}{|c|}{$\mathrm{Ts}=23, \mathrm{O}^{\circ} \mathrm{C}$} \\
\hline HR & \multicolumn{3}{|c|}{$\mathrm{HR}=50 \%$} \\
\hline \multicolumn{4}{|c|}{ Condiciones interiores locales no acondicionados } \\
\hline & Invierno & Verano & Anual \\
\hline Temperatura & $\mathrm{Ts}=7,0^{\circ} \mathrm{C}$ & $\mathrm{Ts}=21^{\circ} \mathrm{C}$ & $\mathrm{Ts}=13,7^{\circ} \mathrm{C}$ \\
\hline HR & $\mathrm{HR}=65 \%$ & $\mathrm{HR}=46 \%$ & $\mathrm{HR}=57 \%$ \\
\hline
\end{tabular}
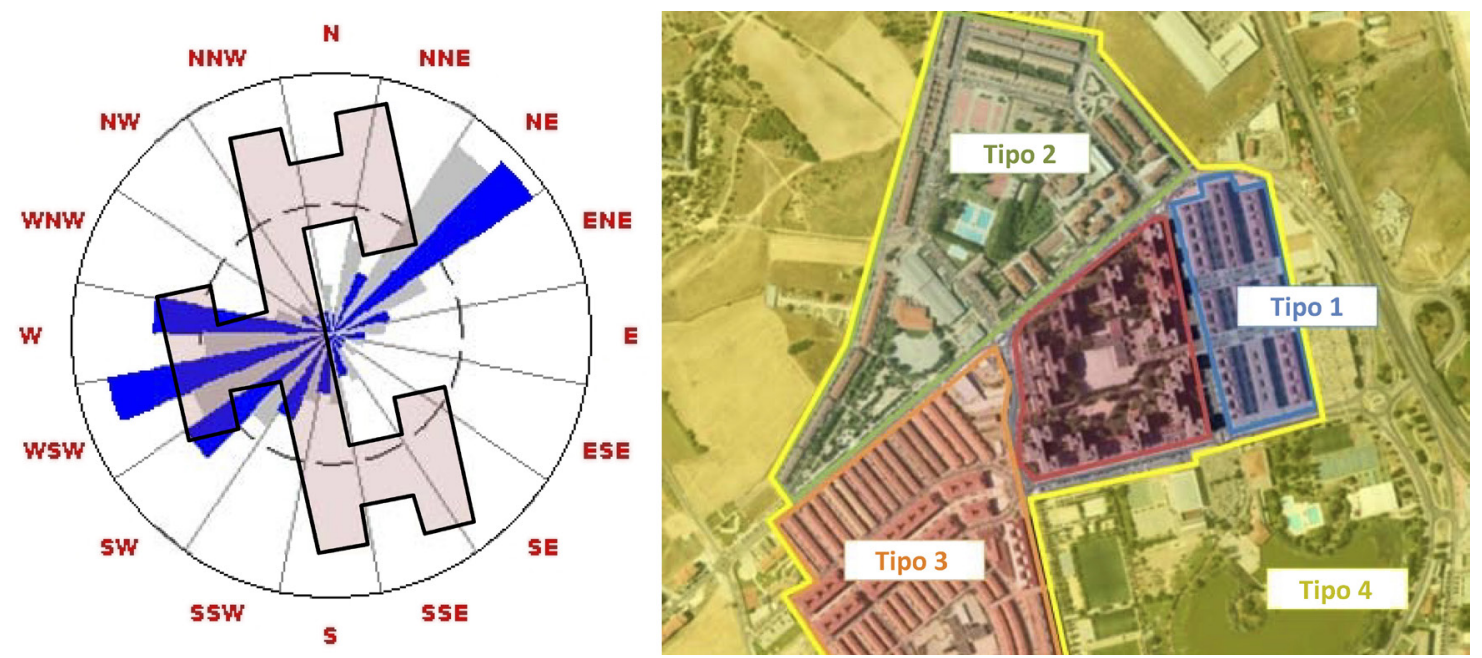

Figura 3. Entorno eólico y acción sobre el edificio.

neutra. En invierno (asumiendo que $\mathrm{T}_{\mathrm{INT}}>\mathrm{T}_{\mathrm{EXT}}$ ), $\Delta p$ provocará, por encima de esta altura, una salida y, por debajo, una entrada de aire al edificio. La ecuación que expresa la diferencia de presión entre el nivel inferior y superior $(H=$ altura superior del espacio vertical) de un edificio puede expresarse:

$$
\Delta p=\left(\rho_{o}-\rho_{i}\right) \cdot g \cdot H
$$

En los edificios de Torrelago este proceso se desarrolla a través de los núcleos verticales integrados en el edificio (ascensores, núcleos de escaleras, patinillos, etc.) pudiéndose expresar el gradiente total sobre la envolvente exterior ( $\mathrm{Fi}$ gura 4).

\subsection{Presiones eólicas}

La acción del viento es uno de los fenómenos físicos más complejos de simular. El elevado número de variables que intervienen en su definición hace imposible tener en consideración todos los parámetros que condicionan su compor- tamiento en el entorno urbano. De particular importancia es dar la mejor aproximación posible a las características fluidodinámicas del aire y a las características geométricas y de rugosidad del entorno. El ámbito de actuación adopta un régimen turbulento, dada la existencia de obstáculos, la viscosidad del aire, la velocidad del fluido y las características del medio (9) (10) (11).

El estudio experimental del viento, basado en casos reales y modelos a escala en túneles de viento (12), ha permitido acercar su comportamiento a modelos predictivos, que son los simulados mediante CFD. Este trabajo adopta el modelo Reynolds Average Navier-Stokes (RANS) como el más adecuado para la simulación de procesos estacionarios y promediados en el tiempo, con la obtención del comportamiento del flujo en los entornos urbanos (13).

Los datos empíricos demuestran (14) que se ha de discernir entre el modelo de flujo paralelo a los cerramientos frente a los modelos perpendicular u oblicuo. En los casos en los que 


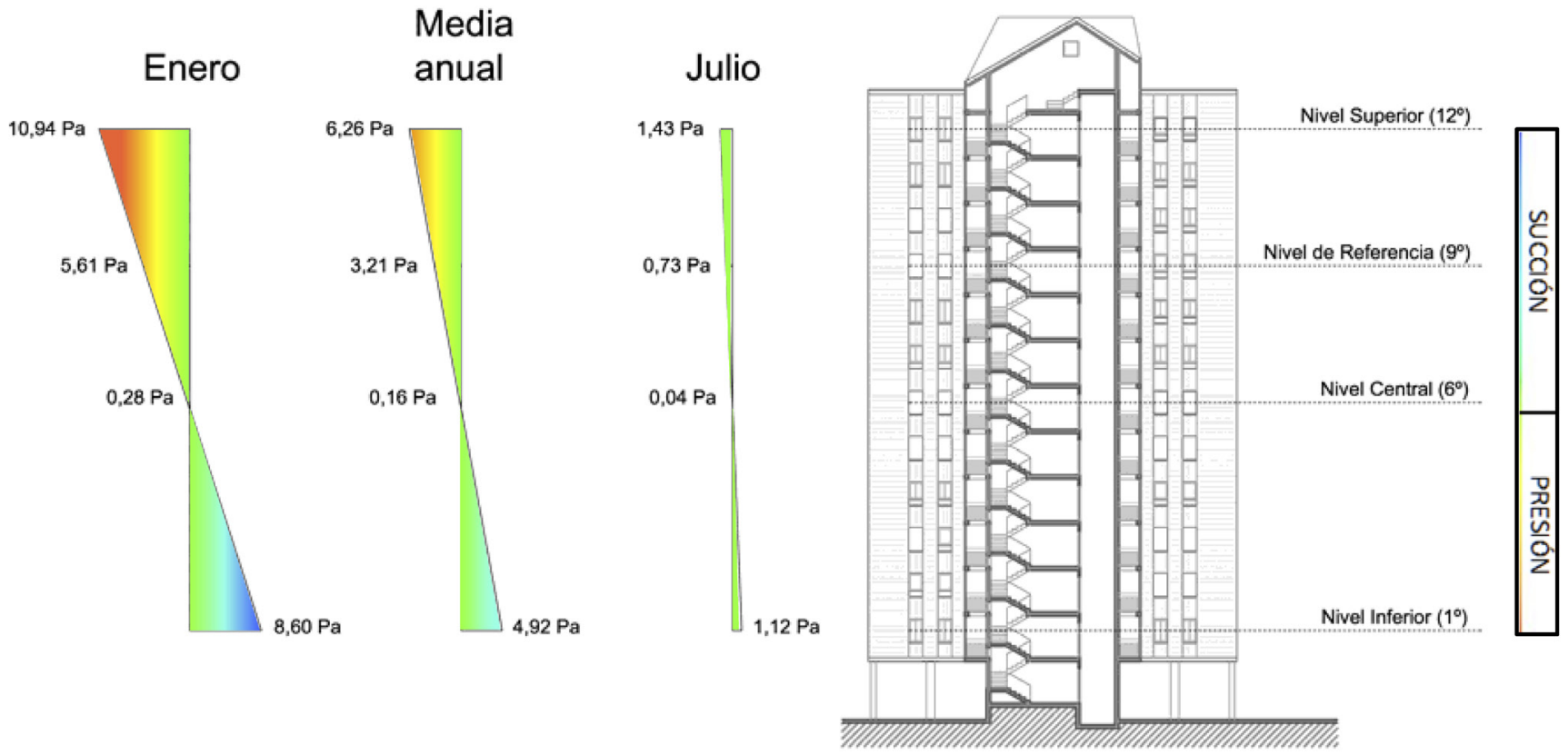

Figura 4. Gradiente de presiones producto de las temperaturas.

la acción del viento tenga una componente axial al corredor por donde discurre, su perfil de velocidades se comportará siguiendo una ecuación logarítmica:

$$
U=\frac{U^{*}}{\kappa} \ln \left(\frac{z-z_{d}}{z_{0}}\right)
$$

Siendo: $z$ la dimensión vertical desde la cota del suelo, $U$ la velocidad del viento a la cota $z, U^{*}$ la velocidad de fricción, $\kappa$ la constante de Von Karman $(\kappa \approx 0,41), z_{d}$ el desplazamiento vertical del plano de rozamiento, $z_{0}$ la altura de rugosidad.

Por el contrario, los modelos perpendicular y oblicuo requerirán de un estudio pormenorizado del movimiento del aire a través del perfil urbano. Los algoritmos de este modelo consideran al aire como un fluido perfecto, y por tanto incompresible:

$$
u_{p}=U_{o} \cdot \exp \left(\frac{y \cdot z_{0}}{0,1 \cdot h_{b}^{2}}\right)
$$

Siendo: $u_{p}$ la velocidad horizontal del viento para cotas inferiores a la coronación de los edificios, $U_{0}$ la velocidad constante de referencia a nivel de suelo, $h_{b}^{2}$ la altura de la edificación anexa al corredor urbano, $z_{\mathrm{o}}$ la altura aerodinámica de rugosidad que define la cota donde la velocidad del viento se hace nula (15).

El dominio de la simulación cumple los requisitos dimensionales definidos por otros investigadores (16), con una distancia desde el origen de coordenadas de $8 \mathrm{H}$ (en el eje de simetría del modelo a calcular) a las paredes (laterales y superior), y de $5 \mathrm{H}$ y $15 \mathrm{H}$ longitudinalmente aguas arriba y aguas abajo del modelo, siendo $\mathrm{H}$ la altura del edificio que sirve de obstáculo. Para este dominio ideal de modelización se diseña una malla hexaédrica tridimensional, con un número de $\approx 6 \cdot 10^{6}$ celdas para el bloque aislado y de $\approx 3 \cdot 10^{6}$ para la urbanización. Para el modelo de bloques individuales, las celdas reducen su tamaño de forma progresiva en relación a la proximidad de los volúmenes sólidos, con una variación entre contiguas inferior al $10 \%$. Así la celda de menor tamaño tiene una dimensión de 0,006 H (o,25 m), que cumple con el estándar establecido (17) para todas aquellas ubicadas en la subcapa viscosa. La separación entre nodos se ha fijado para hacerlos coincidir con los puntos de lectura de presión eólica sobre fachadas, a una distancia de 0,036 H (1,50 m).

La necesaria validación de todo estudio numérico debe tener en cuenta las diferencias sistemáticas entre los ensayos de campo, aquellos de túnel de viento y la simulación computacional. Las experiencias de campo representan medias de intervalos de hasta 30 minutos debido a la variabilidad meteorológica, con una deficiente repetitividad. Las simulaciones CFD responden adecuadamente a soluciones estabilizadas en un tiempo infinito, por lo que deben su validación a datos obtenidos con túnel de viento en condiciones estacionarias.

La simulación numérica, realizada mediante el software $A n$ sys Fluent $R 15 . O^{\odot}$, requirió entonces de la validación de un modelo urbano genérico, que recreara las condiciones existentes en el modelo real, para verificar que los resultados alcanzados con esa configuración se correspondían con los valores obtenidos en las experiencias a escala. Se utilizó los casos ensayados en un túnel de viento BLASIUS (18) dentro del ámbito del proyecto CEDVAL (19), del Windkanallabor EWTL del Meteorologisches Institut, Universität Hamburg. Dado que las mediciones en el interior del túnel de viento aportan datos característicos de velocidad, será ésta la tomada en consideración. Así se han validado las variables intervinientes en la dinámica de los fluidos (perfiles de velocidad y turbulencia $(\mathrm{k}-\varepsilon)$, su comportamiento sobre un edificio aislado, y su aplicación en modelos urbanos complejos. La precisión obtenida en el modelo de edificio aislado y en el modelo urbano se considera satisfactoria para obtener resultados de cálculo aplicables al caso real (Tabla 2) (20).

\subsubsection{Estudio de viento en bloque aislado}

Diez son las variaciones estudiadas del edificio aislado, con distintas orientaciones, estacionalidad, exposición al viento y 
Tabla 2. Precisión de los resultados de los modelos de validación.

\begin{tabular}{|l|c|c|}
\hline \multirow{2}{*}{ Método } & \multicolumn{2}{c|}{$\begin{array}{c}\text { RANS Realizable - Enhanced Wall } \\
\text { Treatment }\end{array}$} \\
\cline { 2 - 3 } Variable & Bloque aislado & Modelo urbano \\
\hline Velocidad & $\pm 1,05 \%$ & $\pm 3,68 \%$ \\
\hline Velocidad X & $\pm 1,14 \%$ & $\pm 2,01 \%$ \\
\hline Velocidad Y & $\pm 12,30 \%$ & $\pm 12,46 \%$ \\
\hline Velocidad Z & $\pm 4,57 \%$ & $\pm 4,23 \%$ \\
\hline
\end{tabular}

agrupación con otros bloques en H. La simulación CFD conseguida proporciona gran cantidad de datos del modelo de flujo: distribuciones de velocidad, presiones estáticas y dinámicas, trayectoria de las partículas, edad del aire, etc. Por ejemplo, el coeficiente de presión eólico sobre los cerramientos permite distinguir la zona sometida a efectos de presión y de succión por la acción del viento, y obtener posteriormente sus valores en Pascales.

Un análisis en detalle obliga a caracterizar estas presiones a distintas alturas del edificio, en correspondencia con el perfil variable de velocidades del viento. Los valores obtenidos sobre los distintos cerramientos que delimitan a cada una de las viviendas establecerán el gradiente de presiones total debido a la acción eólica. El estudio se enfocó en las direcciones del viento WSW y NE, predominantes a lo largo del año, asimilando su acción a una presión de tipo ortogonal sobre las fachadas. En cuanto a las alturas consideradas, se calcularon los valores correspondientes a un nivel inferior ( $1^{\circ}$ ), central $\left(6^{\circ}\right)$, de referencia $\left(9^{\circ}\right)$ y superior $\left(1^{\circ}\right.$ ). Por último, la distinta orientación de los paramentos de una misma vivienda se caracteriza mediante las letras $\alpha, \beta, \gamma$ y $\delta$ (Figura 5).

\subsubsection{Análisis del modelo urbano}

La simulación a nivel de distrito permite identificar aquellos elementos que interceptan el libre fluir del viento, alterando el desplazamiento del aire a las regiones ubicadas aguas abajo. En este aspecto hay que destacar que la influencia de corredores, áreas abiertas y pantallas de edificios obligan siempre a realizar un estudio específico que cuantifique la merma de ventilación natural en cada edificio, respecto a considerarlos como entidades aisladas.

En este estudio se utilizaron los valores medios anuales del viento, actuando según las direcciones predominantes WSW y NE (Figura 6).

\subsection{Permeabilidad de los cerramientos}

Los ensayos de presurización en viviendas permiten determinar la permeabilidad de la envolvente, pudiéndose discriminar el valor de partes ciegas y huecos. El ensayo, realizado según la Norma Europea EN13829 y denominado comúnmente Blower Door Test, crea un diferencial de presiones en el interior de la zona a ensayar respecto al exterior, midiéndose el flujo de aire que circula a través de un ventilador. En una primera fase, el ensayo se realiza mediante el método $B$ de la norma (ensayo de la envolvente del edificio), con el sellado progresivo de los puntos de filtración más significativos (carpinterías, cajas de persianas) para establecer la incidencia que tienen en la permeabilidad del cerramiento. Posteriormente se aplica el método A (ensayo de un edificio en uso) para verificar el estado de los shunts comunitarios de ventilación. En total se realizaron 14 ensayos de presurización/despresurización en otras tantas viviendas, a la vista de cumplir la recomendación de ensayar el mayor número posible de ellas, comprendiendo al menos un ático, una de la cota más baja, y pisos intermedios.

El caudal de filtración obtenido en el ensayo se mide en función del gradiente de presión a través del cerramiento de la vivienda, adoptando una ley potencial:

$$
Q_{\Delta P}=C_{L} \cdot \Delta P^{n}
$$

Siendo: $C_{L}$ el coeficiente de caudal de filtración, $\Delta P$ la diferencia de presión, y $n$ el exponente de la ley potencial. Esta ley nos permite obtener el caudal de filtración equivalente al gradiente de presión deseado, lo que permite cuantificar el aire que penetra a través de cada uno de los paramentos.

Las características constructivas de los bloques hacen suponer que las infiltraciones así calculadas se producen de manera mayoritaria a través de la envolvente exterior:
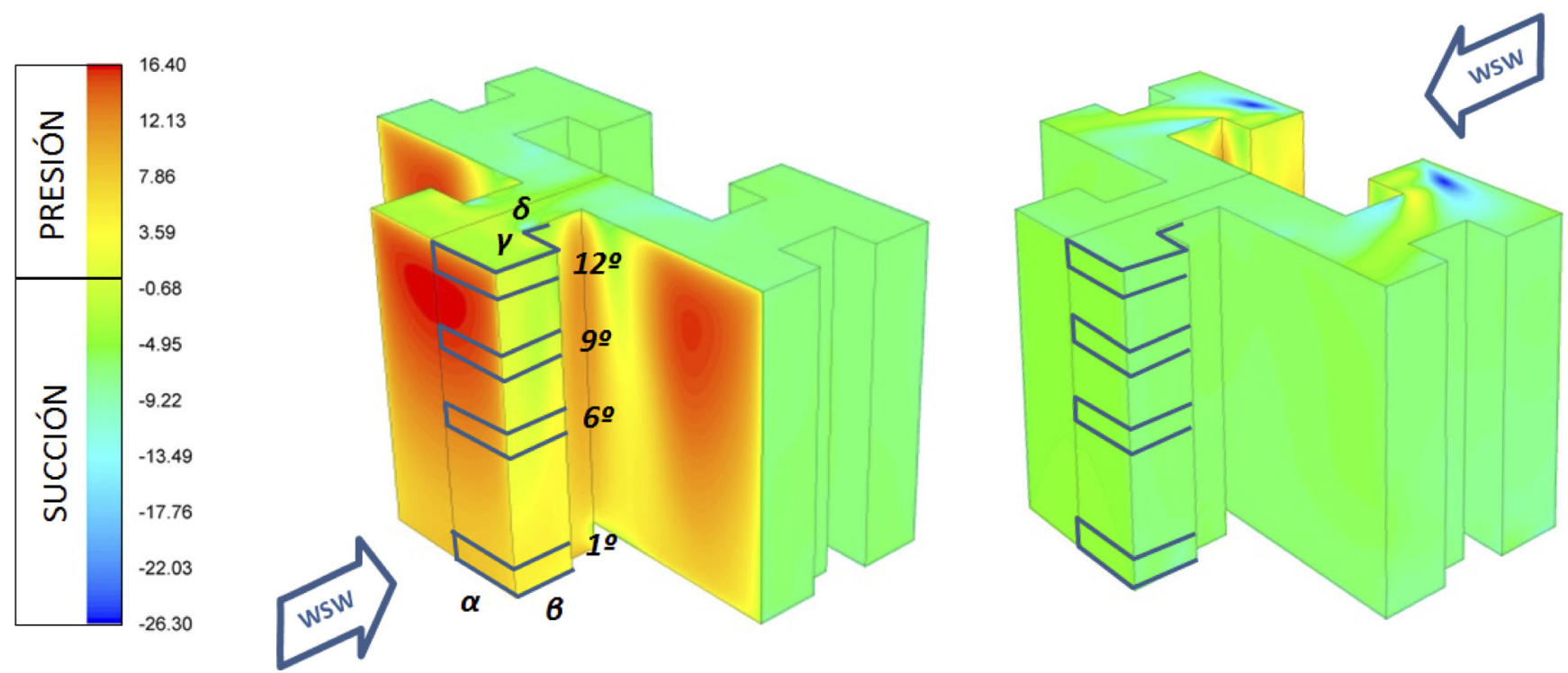

Figura 5. Coeficiente de presión eólico (media anual) sobre los cerramientos. 


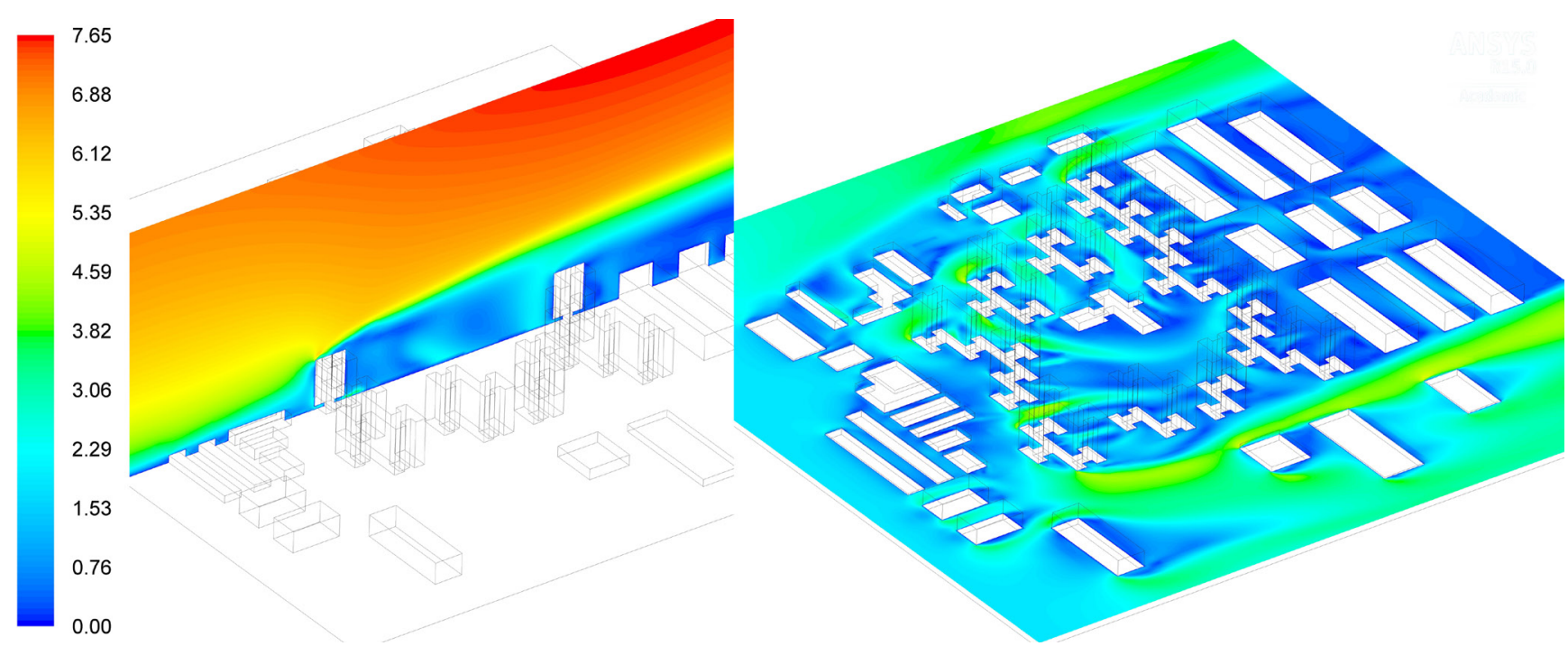

Figura 6. Distribución de la velocidad del viento WSW a nivel de distrito (planta y sección).

- La separación con otras viviendas de su misma planta se materializa mediante cerramientos de tipo medio-pesado, sin elementos comunes que interrumpan dicha continuidad.

- La realización del ensayo utiliza la puerta de acceso a la vivienda para colocar el ventilador, lo que se traduce en el virtual sellado de este punto débil de separación con las zonas comunes del edificio.

- Las instalaciones de saneamiento discurren por la propia vivienda, sin atravesar el forjado. Las conducciones generales verticales utilizan patinillos de fábrica independientes.

- El sellado de todas las carpinterías y de puntos de filtración localizada dio por resultado ensayos con unas tasas prácticamente nulas en el resto del cerramiento.

\subsection{Caudal de ventilación natural}

A partir de los datos anteriores es posible cuantificar el caudal de ventilación natural de cada vivienda, mediante un proceso que analiza de forma conjunta los distintos elementos de la envolvente expuestos al gradiente de presiones. El procedimiento está basado en la Norma UNE-EN 13465:2004 Métodos de cálculo para la obtención del caudal de aire en viviendas, que sigue las pautas de las teorías clásicas de la ventilación natural.

Los sistemas de ecuaciones así planteados requieren la utilización de un software de cálculo, Engineering Equation Solver EES, para determinar el gradiente de presión en cada uno de los cerramientos y el caudal de ventilación natural.

\section{RESULTADOS Y ANÁLISIS DE LA VENTILACIÓN NATURAL}

A nivel de bloque aislado, se puede hablar de gradientes de presión entre o y 12 Pa para los cerramientos expuestos a barlovento y de entre -2 y -6 Pa para aquellos a sotavento. En el análisis del modelo urbano, los resultados corroboran que los edificios más expuestos pueden asimilarse a bloques aislados. A la vez, actúan simultáneamente de pantalla de aquellos otros ubicados aguas abajo, mermando las presiones eólicas que afectan sus cerramientos en un orden del 70 al $90 \%$. Esto significa una reducción del caudal disponible de ventilación natural.
La superposición de presiones producto de temperatura y viento permite calcular su efecto sobre la superficie de cada uno de los cerramientos que envuelve las viviendas (Tabla 3). El efecto resultante provoca distintas casuísticas a lo largo del año, con alternancia de presiones y succiones estacionales en los pisos altos y bajos.

La campaña de ensayos permitió conocer las condiciones iniciales del proyecto, así como las intervenciones particulares de los propietarios, en particular en lo que se refiere a la sustitución de carpinterías y al taponamiento de shunts comunitarios de ventilación. En concordancia con el estudio de viento del bloque aislado, en cuanto a la caracterización de las presiones eólicas a distintas alturas del edificio, se recabaron datos en los ensayos de presurización de las viviendas correspondientes a un nivel inferior $\left(1^{\circ}\right)$, central $\left(6^{\circ}\right)$, de referencia (9) y superior (12ㅇ) (Tabla 4 ).

Dado que fue posible caracterizar una vivienda sin modificar, acorde al proyecto original $\left(9^{\circ} \mathrm{C}\right)$, se adopta esta configuración de referencia, como punto de base de las posteriores comparaciones.

Se simula así el caudal de ventilación natural en viviendas de un bloque, a partir de su altura en el edificio, las temperaturas ambientales, la exposición a los vientos dominantes, y una configuración constructiva acorde al proyecto original. Las renovaciones de aire por hora $(\mathrm{ACH})$ obtenidas varían, como era de suponer, en función de la distinta altura de la vivienda en el edificio y de las condiciones climáticas (Figura 7).

Sin embargo, las alteraciones constructivas han cambiado esta configuración inicial, pudiéndose simular las consecuencias de dichos cambios a partir de los datos obtenidos en los ensayos de presurización. Se puede calcular el comportamiento a las distintas alturas de una misma configuración de vivienda con distinto número de conductos verticales de ventilación (2,1 o ninguno) o distintas tipos de carpinterías (Figura 8). A partir de este punto es posible caracterizar cada vivienda de los bloques del distrito, aplicándoseles la reducción correspondiente de la presión eólica sobre sus cerramientos.

Se observa que los valores mínimos anuales establecidos en el DB-HS3 sólo se alcanzan en viviendas de 4 dormi- 
Tabla 3. Distribución de presiones por cerramiento según la altura (fachada a barlovento).

\begin{tabular}{|c|c|c|c|c|c|c|c|c|c|c|c|}
\hline & \multirow{2}{*}{ Vivienda } & \multirow{2}{*}{ Cerr. } & \multicolumn{3}{|c|}{ Efecto térmico } & \multicolumn{3}{|c|}{ Efecto eólico } & \multicolumn{3}{|c|}{ Total combinado } \\
\hline & & & Invierno & Verano & Anual & Invierno & Verano & Anual & Invierno & Verano & Anual \\
\hline \multirow{17}{*}{ 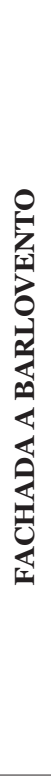 } & \multirow{4}{*}{ 1ㅇ (inferior) } & $\alpha$ & \multirow{4}{*}{$8,60 \mathrm{~Pa}$} & \multirow{4}{*}{$1,12 \mathrm{~Pa}$} & \multirow{4}{*}{ 4,92 Pa } & $7,28 \mathrm{~Pa}$ & $5,32 \mathrm{~Pa}$ & $7,83 \mathrm{~Pa}$ & $15,88 \mathrm{~Pa}$ & $6,44 \mathrm{~Pa}$ & $12,95 \mathrm{~Pa}$ \\
\hline & & $\beta$ & & & & $3,12 \mathrm{~Pa}$ & $2,29 \mathrm{~Pa}$ & $2,76 \mathrm{~Pa}$ & $11,72 \mathrm{~Pa}$ & $3,41 \mathrm{~Pa}$ & $7,68 \mathrm{~Pa}$ \\
\hline & & $\gamma$ & & & & $4,87 \mathrm{~Pa}$ & $3,56 \mathrm{~Pa}$ & $6,22 \mathrm{~Pa}$ & $13,47 \mathrm{~Pa}$ & 4,68 Pa & $11,14 \mathrm{~Pa}$ \\
\hline & & $\delta$ & & & & $4,84 \mathrm{~Pa}$ & $3,54 \mathrm{~Pa}$ & $6,10 \mathrm{~Pa}$ & $13,44 \mathrm{~Pa}$ & 4,66 Pa & $11,02 \mathrm{~Pa}$ \\
\hline & \multirow{4}{*}{60 (central) } & $a$ & \multirow{4}{*}{$0,28 \mathrm{~Pa}$} & \multirow{4}{*}{ 0,04 Pa } & \multirow{4}{*}{$0,16 \mathrm{~Pa}$} & 9,72 $\mathrm{Pa}$ & 7,14 Pa & 9,26 Pa & $10,00 \mathrm{~Pa}$ & $7,18 \mathrm{~Pa}$ & $9,42 \mathrm{~Pa}$ \\
\hline & & $\beta$ & & & & $1,56 \mathrm{~Pa}$ & $1,16 \mathrm{~Pa}$ & $2,26 \mathrm{~Pa}$ & $1,84 \mathrm{~Pa}$ & $1,20 \mathrm{~Pa}$ & $2,42 \mathrm{~Pa}$ \\
\hline & & $\gamma$ & & & & $5,16 \mathrm{~Pa}$ & $3,76 \mathrm{~Pa}$ & $6,97 \mathrm{~Pa}$ & $5,44 \mathrm{~Pa}$ & $3,80 \mathrm{~Pa}$ & $7,13 \mathrm{~Pa}$ \\
\hline & & $\delta$ & & & & $5,39 \mathrm{~Pa}$ & $3,93 \mathrm{~Pa}$ & $6,87 \mathrm{~Pa}$ & $5,67 \mathrm{~Pa}$ & $3,97 \mathrm{~Pa}$ & $7,03 \mathrm{~Pa}$ \\
\hline & \multirow{4}{*}{ 9으 (referencia) } & $\alpha$ & \multirow{4}{*}{$-5,61 \mathrm{~Pa}$} & \multirow{4}{*}{ 0,73 Pa } & \multirow{4}{*}{$-3,21 \mathrm{~Pa}$} & $10,90 \mathrm{~Pa}$ & $8,05 \mathrm{~Pa}$ & 9,91 Pa & $5,29 \mathrm{~Pa}$ & $8,78 \mathrm{~Pa}$ & $6,70 \mathrm{~Pa}$ \\
\hline & & $\beta$ & & & & $0,60 \mathrm{~Pa}$ & $0,45 \mathrm{~Pa}$ & $1,72 \mathrm{~Pa}$ & $-5,01 \mathrm{~Pa}$ & $1,18 \mathrm{~Pa}$ & $-1,49 \mathrm{~Pa}$ \\
\hline & & $\gamma$ & & & & $5,12 \mathrm{~Pa}$ & $3,72 \mathrm{~Pa}$ & $6,74 \mathrm{~Pa}$ & $-0,49 \mathrm{~Pa}$ & $4,45 \mathrm{~Pa}$ & $3,73 \mathrm{~Pa}$ \\
\hline & & $\delta$ & & & & $5,55 \mathrm{~Pa}$ & $4,03 \mathrm{~Pa}$ & $6,94 \mathrm{~Pa}$ & $-0,06 \mathrm{~Pa}$ & $4,76 \mathrm{~Pa}$ & $3,73 \mathrm{~Pa}$ \\
\hline & \multirow{4}{*}{12 o (superior) } & $\alpha$ & \multirow{4}{*}{$-10,94 \mathrm{~Pa}$} & \multirow{4}{*}{$1,43 \mathrm{~Pa}$} & \multirow{4}{*}{$-6,26 \mathrm{~Pa}$} & $11,64 \mathrm{~Pa}$ & $5,58 \mathrm{~Pa}$ & $10,20 \mathrm{~Pa}$ & $0,70 \mathrm{~Pa}$ & 7,01 $\mathrm{Pa}$ & $3,94 \mathrm{~Pa}$ \\
\hline & & $\beta$ & & & & $-0,02 \mathrm{~Pa}$ & $-0,03 \mathrm{~Pa}$ & $1,21 \mathrm{~Pa}$ & $-10,96 \mathrm{~Pa}$ & $1,40 \mathrm{~Pa}$ & $-5,05 \mathrm{~Pa}$ \\
\hline & & $\gamma$ & & & & $4,81 \mathrm{~Pa}$ & $3,48 \mathrm{~Pa}$ & $6,67 \mathrm{~Pa}$ & $-6,13 \mathrm{~Pa}$ & 4,91 Pa & $0,41 \mathrm{~Pa}$ \\
\hline & & $\delta$ & & & & $5,29 \mathrm{~Pa}$ & $3,83 \mathrm{~Pa}$ & $6,89 \mathrm{~Pa}$ & $-5,65 \mathrm{~Pa}$ & $5,26 \mathrm{~Pa}$ & $0,63 \mathrm{~Pa}$ \\
\hline & \multicolumn{2}{|l|}{ Cubierta } & $-10,94 \mathrm{~Pa}$ & 1,43 $\mathrm{Pa}$ & $-6,26 \mathrm{~Pa}$ & $-2,36 \mathrm{~Pa}$ & $-1,77 \mathrm{~Pa}$ & $-0,66 \mathrm{~Pa}$ & $-13,30 \mathrm{~Pa}$ & $-0,34 \mathrm{~Pa}$ & $-6,92 \mathrm{~Pa}$ \\
\hline \multirow{17}{*}{ 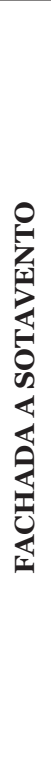 } & \multirow{4}{*}{19 (inferior) } & $a$ & & & & $-3,19 \mathrm{~Pa}$ & $-3,71 \mathrm{~Pa}$ & $-2,85 \mathrm{~Pa}$ & $5,41 \mathrm{~Pa}$ & $-2,59 \mathrm{~Pa}$ & 2,07 Pa \\
\hline & & $\beta$ & $8.60 \mathrm{~Pa}$ & $112 \mathrm{~Pa}$ & $402 \mathrm{~Pa}$ & $-4,50 \mathrm{~Pa}$ & $-5,65 \mathrm{~Pa}$ & $-3,95 \mathrm{~Pa}$ & $4,10 \mathrm{~Pa}$ & $-4,53 \mathrm{~Pa}$ & $0,97 \mathrm{~Pa}$ \\
\hline & & $\gamma$ & $0,001 \mathrm{a}$ & $1,121 \mathrm{a}$ & $4,921 \mathrm{a}$ & $-4,14 \mathrm{~Pa}$ & $-5,42 \mathrm{~Pa}$ & $-3,69 \mathrm{~Pa}$ & $4,46 \mathrm{~Pa}$ & $-4,30 \mathrm{~Pa}$ & 1,23 $\mathrm{Pa}$ \\
\hline & & $\delta$ & & & & $-4,21 \mathrm{~Pa}$ & $-5,43 \mathrm{~Pa}$ & $-3,80 \mathrm{~Pa}$ & $4,39 \mathrm{~Pa}$ & $-4,31 \mathrm{~Pa}$ & $1,12 \mathrm{~Pa}$ \\
\hline & & $\alpha$ & & & & $-3,41 \mathrm{~Pa}$ & $-3,82 \mathrm{~Pa}$ & $-3,04 \mathrm{~Pa}$ & $-3,13 \mathrm{~Pa}$ & $-3,78 \mathrm{~Pa}$ & $-2,88 \mathrm{~Pa}$ \\
\hline & $60($ contral) & $\beta$ & $\mathrm{O}_{0} \mathrm{P}_{3}$ & $\mathrm{P}_{2}$ & & $-4,41 \mathrm{~Pa}$ & $-5,67 \mathrm{~Pa}$ & $-3,98 \mathrm{~Pa}$ & $-4,13 \mathrm{~Pa}$ & $-5,63 \mathrm{~Pa}$ & $-3,82 \mathrm{~Pa}$ \\
\hline & o= (central) & $\gamma$ & $0,20 \mathrm{Pd}$ & $0,04 \mathrm{Pd}$ & $0,10 \mathrm{Pd}$ & $-4,18 \mathrm{~Pa}$ & $-5,32 \mathrm{~Pa}$ & $-3,78 \mathrm{~Pa}$ & $-3,90 \mathrm{~Pa}$ & $-5,28 \mathrm{~Pa}$ & $-3,62 \mathrm{~Pa}$ \\
\hline & & $\delta$ & & & & $-4,18 \mathrm{~Pa}$ & $-5,31 \mathrm{~Pa}$ & $-3,77 \mathrm{~Pa}$ & $-3,90 \mathrm{~Pa}$ & $-5,27 \mathrm{~Pa}$ & $-3,61 \mathrm{~Pa}$ \\
\hline & & $\alpha$ & & & & $-3,39 \mathrm{~Pa}$ & $-3,85 \mathrm{~Pa}$ & $-3,04 \mathrm{~Pa}$ & $-9,00 \mathrm{~Pa}$ & $-3,12 \mathrm{~Pa}$ & $-6,25 \mathrm{~Pa}$ \\
\hline & oo (referencia) & $\beta$ & $-5.61 \mathrm{~Pa}$ & $0.73 \mathrm{~Pa}$ & $-321 \mathrm{~Pa}$ & $-4,38 \mathrm{~Pa}$ & $-5,69 \mathrm{~Pa}$ & $-3,96 \mathrm{~Pa}$ & $-9,99 \mathrm{~Pa}$ & $-4,96 \mathrm{~Pa}$ & $-7,17 \mathrm{~Pa}$ \\
\hline & 9=(генегепста) & $\gamma$ & $-5,01 \mathrm{~Pa}$ & $0, / 3 \mathrm{~Pa}$ & $-3,21 \mathrm{Fa}$ & $-4,17 \mathrm{~Pa}$ & $-5,32 \mathrm{~Pa}$ & $-3,77 \mathrm{~Pa}$ & $-9,78 \mathrm{~Pa}$ & $-4,59 \mathrm{~Pa}$ & $-6,98 \mathrm{~Pa}$ \\
\hline & & $\delta$ & & & & $-4,16 \mathrm{~Pa}$ & $-5,31 \mathrm{~Pa}$ & $-3,76 \mathrm{~Pa}$ & $-9,77 \mathrm{~Pa}$ & $-4,58 \mathrm{~Pa}$ & $-6,97 \mathrm{~Pa}$ \\
\hline & & $a$ & & & & $-3,33 \mathrm{~Pa}$ & $-3,99 \mathrm{~Pa}$ & $-3,01 \mathrm{~Pa}$ & $-14,27 \mathrm{~Pa}$ & $-2,56 \mathrm{~Pa}$ & $-9,27 \mathrm{~Pa}$ \\
\hline & 100 & $\beta$ & $-1004 \mathrm{P}_{9}$ & $14 \mathrm{Pr}_{9}$ & & $-4,39 \mathrm{~Pa}$ & $-5,67 \mathrm{~Pa}$ & $-3,96 \mathrm{~Pa}$ & $-15,33 \mathrm{~Pa}$ & $-4,24 \mathrm{~Pa}$ & $-10,22 \mathrm{~Pa}$ \\
\hline & $12-($ superior $)$ & $\gamma$ & $-10,94 \mathrm{ra}$ & $1,43 \mathrm{ra}$ & $-0,20 \mathrm{ra}$ & $-4,19 \mathrm{~Pa}$ & $-5,53 \mathrm{~Pa}$ & $-3,78 \mathrm{~Pa}$ & $-15,13 \mathrm{~Pa}$ & $-4,10 \mathrm{~Pa}$ & $-10,04 \mathrm{~Pa}$ \\
\hline & & $\delta$ & & & & $-4,17 \mathrm{~Pa}$ & $-5,53 \mathrm{~Pa}$ & $-3,77 \mathrm{~Pa}$ & $-15,11 \mathrm{~Pa}$ & $-4,10 \mathrm{~Pa}$ & $-10,03 \mathrm{~Pa}$ \\
\hline & Cubierta & & $-10,94 \mathrm{~Pa}$ & 1,43 Pa & $-6,26 \mathrm{~Pa}$ & $-4,44 \mathrm{~Pa}$ & $-3,53 \mathrm{~Pa}$ & $-4,01 \mathrm{~Pa}$ & $-15,38 \mathrm{~Pa}$ & $-2,10 \mathrm{~Pa}$ & $-10,27 \mathrm{~Pa}$ \\
\hline
\end{tabular}

Tabla 4. Resultados de los ensayos de presurización.

\begin{tabular}{|c|c|c|c|c|c|}
\hline Vivienda & $\begin{array}{c}\text { Tasa } n_{50} \\
(\mathbf{1} / \mathbf{h})\end{array}$ & $\begin{array}{l}\text { Exponente } \\
\mathbf{n}\end{array}$ & $\begin{array}{l}\text { Permeabilidad } \\
\text { al aire de la } \\
\text { carpintería } \\
\text { a 5o Pa } \\
\left(\mathbf{m}^{3} / \mathbf{h} \cdot \mathbf{m}^{2}\right)\end{array}$ & $\begin{array}{l}\text { Disponibilidad } \\
\text { de conducto } \\
\text { vertical }\end{array}$ & Notas \\
\hline $\begin{array}{l}\text { Bloque } 9 \\
\text { Piso12D }\end{array}$ & 2,8 & 0,61 & $\begin{array}{c}3,0 \\
\text { (Clase 3) }\end{array}$ & $\begin{array}{c}\text { Sí } \\
\text { (Cocina y Baño) }\end{array}$ & $\begin{array}{l}\text { Sustitución por carpinterías de PVC abatible y vidrio } \\
\text { doble } \\
\text { Rehabilitación parcial de la envolvente opaca exterior }\end{array}$ \\
\hline $\begin{array}{l}\text { Bloque } 25 \\
\text { Piso 9C }\end{array}$ & 6,2 & 0,64 & $\begin{array}{c}48,6 \\
\text { (Clase o) }\end{array}$ & $\begin{array}{c}\text { Sí } \\
\text { (Cocina y Baño) }\end{array}$ & $\begin{array}{l}\text { Vivienda sin modificar, según el proyecto original } \\
\text { (carpintería corredera de aluminio de } 2 \text { hojas y vidrio } \\
\text { sencillo) }\end{array}$ \\
\hline $\begin{array}{l}\text { Bloque } 6 \\
\text { Piso 6D }\end{array}$ & 3,2 & 0,64 & $\begin{array}{c}8,6 \\
\text { (Clase 2) }\end{array}$ & No & $\begin{array}{l}\text { Sustitución por carpinterías de PVC abatible y vidrio } \\
\text { doble } \\
\text { Rehabilitación parcial de la envolvente opaca exterior }\end{array}$ \\
\hline $\begin{array}{l}\text { Bloque } 4 \\
\text { Piso 1D }\end{array}$ & 3,8 & 0,66 & $\begin{array}{c}15,0 \\
\text { (Clase 1) }\end{array}$ & $\begin{array}{c}\text { Sí } \\
\text { (Cocina) } \\
\text { No } \\
\text { (Baño) }\end{array}$ & Sustitución por carpinterías correderas de aluminio \\
\hline
\end{tabular}


torios, entre las plantas $1^{\text {a }}$ y $5^{\text {a }}$ del edificio. Incluso estos valores sólo son válidos para los edificios más expuestos, puesto que en la urbanización tendremos otros con mermas en los caudales de hasta el $90 \%$, por el efecto pantalla urbano antes mencionado. Este incumplimiento de la exigencia de calidad del aire obliga a implementar medidas adicionales.
De cara al estudio energético, sin profundizar puesto que excede el marco de este trabajo, habría que incorporar al cálculo de la demanda los caudales de aire no acondicionado específicos de cada vivienda. Subrayar también que esto pone en cuestión el criterio de cálculo simplificado $\left(\mathrm{ACH}=\mathrm{n}_{50} / 20(21)\right)$ que relaciona la tasa $\mathrm{n}_{50}$ de los ensayos de presurización con el caudal anual de infiltración.

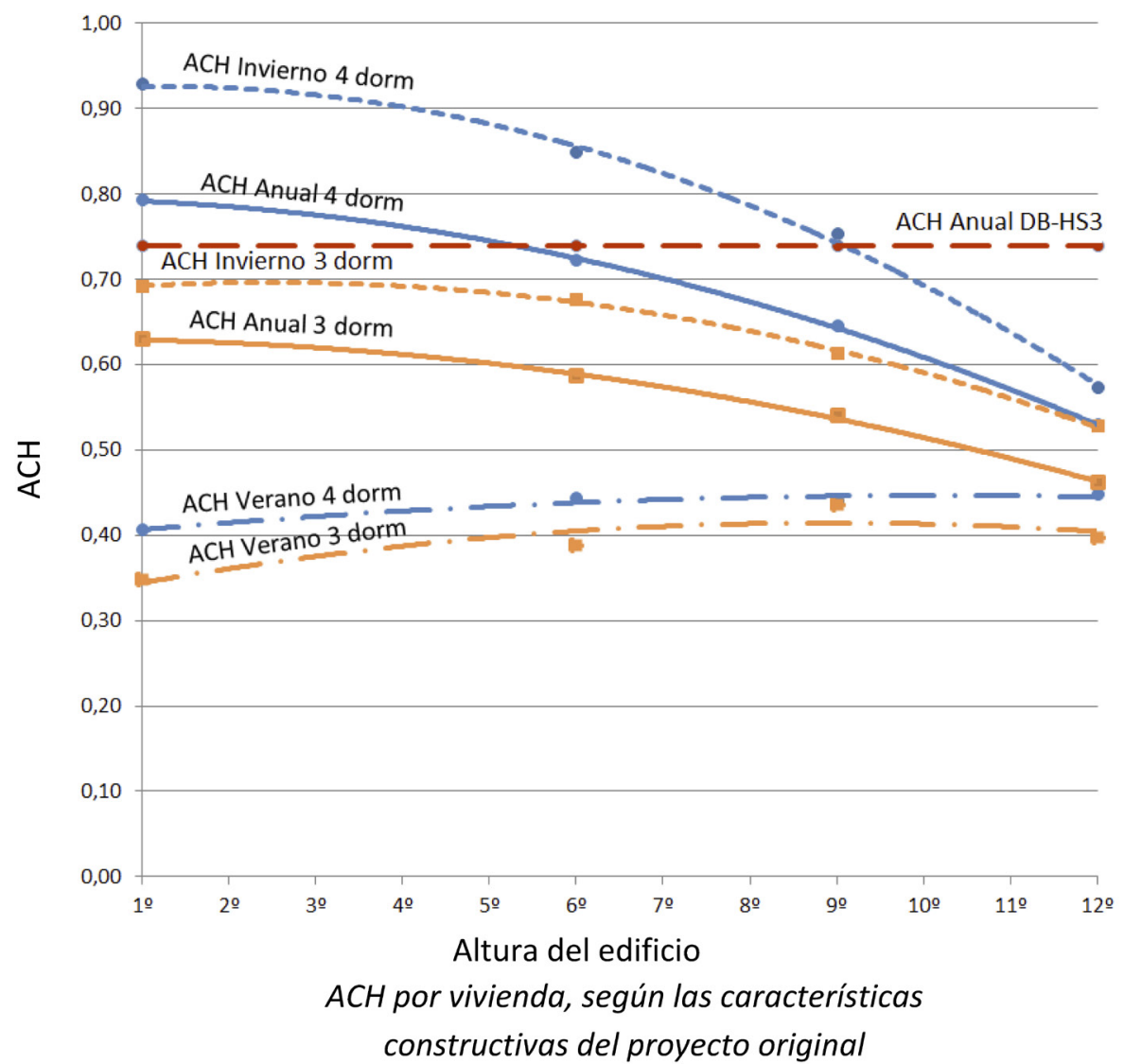

Figura 7. Influencia de la altura de la vivienda y de las condiciones climáticas.
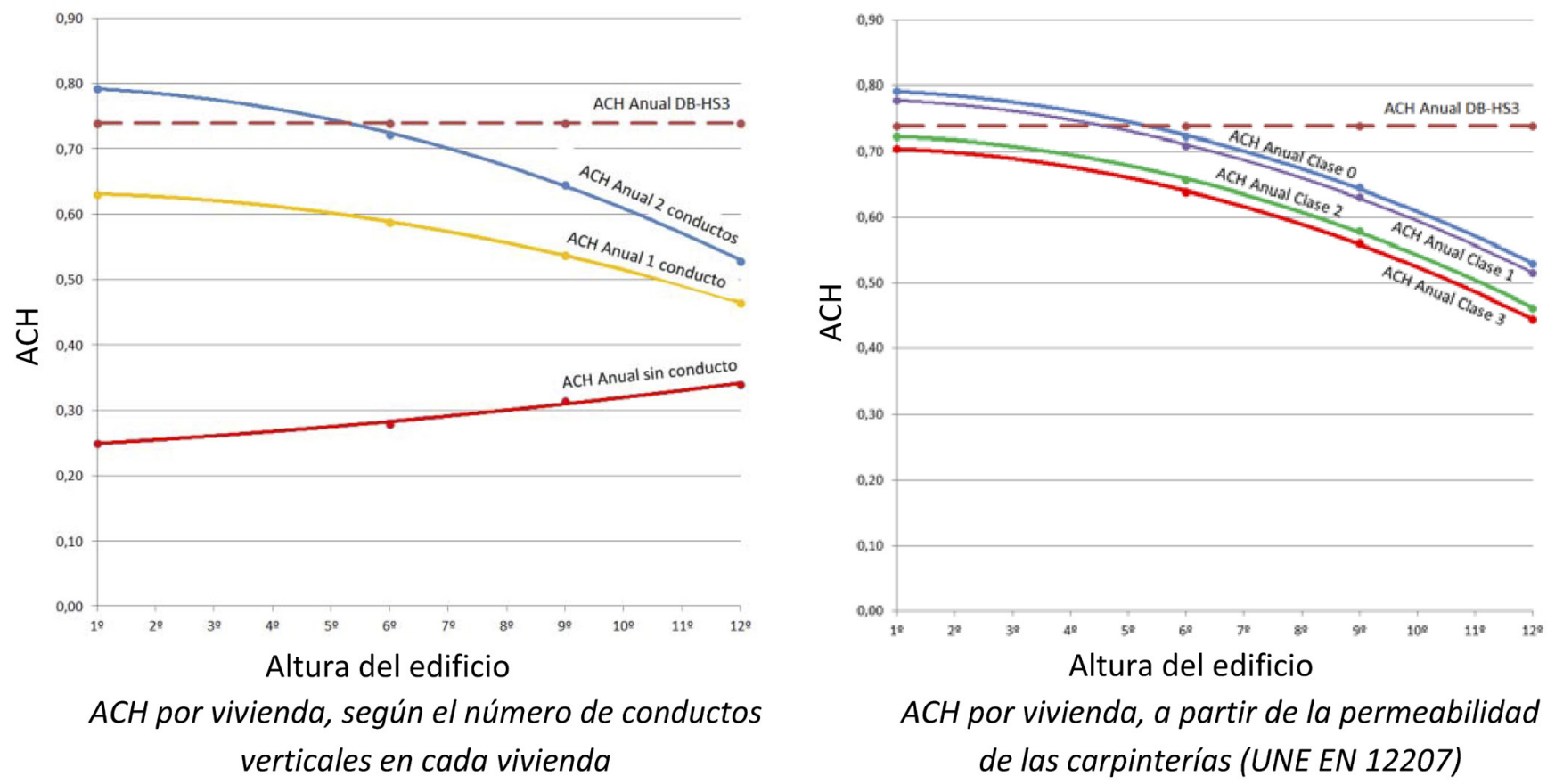

Figura 8. Influencia de distintas alteraciones constructivas. 


\section{IMPLEMENTACIÓN DE LA EXIGENCIA DE CALIDAD DEL AIRE DEL CTE}

Si nos atenemos a las soluciones contempladas en el DB-HS3 caben dos opciones: ventilación híbrida o mecánica, en ambos casos con necesidad de conductos comunes de extracción. El procedimiento menos traumático sería colocar los conductos en fachada, recogiendo los caudales procedentes de los cuartos húmedos de la vivienda (Figura 9). La admisión de aire se haría a través de las propias carpinterías exteriores pudiéndose implementar un sencillo mecanismo de «micro-ventilación» en su manilla de apertura. Habría que añadir que la ventilación híbrida no puede aplicarse al tipo de vivienda de cuatro dormitorios porque su aseo interior requiere un ramal horizontal de extracción solo compatible con la mecánica.

El sistema propuesto en cumplimiento literal del DB HS3 es perfectamente verosímil gracias a las facilidades constructivas del caso que nos ocupa, como son el hecho de que sea una edificación abierta y de que es una implementación simultánea en todo el bloque.

La mejor solución para la rehabilitación, en general, pasaría por aprovechar la aportación de ventilación natural para cada vivienda, constatada en el caso en estudio. Esto se lograría implementando un sistema individualizado controlado por sensores de calidad del aire, que en el peor de los casos accionaría una extracción mecánica.

\section{CONCLUSIONES: EL DB HS-3 EN LA REHABILITACIÓN DE VIVIENDAS}

Primero se plantea una metodología que combina distintas herramientas para estimar justificadamente la tasa de ventilación natural, dado que los métodos simplificados se revelan poco aplicables a simulaciones complejas, al partir de supuestos muy básicos: asunción de presiones uniformes del viento, estacionalidad, simplificación de la volumetría edificatoria, etc. Esta metodología es recreable en otros casos de estudio dado que:

- Los datos climáticos son datos oficiales y estables en el tiempo.

- La configuración CFD se valida mediante datos experimentales de túnel de viento, pudiéndose aplicar para simular con fiabilidad la acción del viento sobre entornos urbanos en condiciones estacionales.

- La combinación de las presiones eólicas y térmicas, éstas producto de las condiciones singulares de temperatura, aporta el gradiente total sobre cada uno de los cerramientos, según su altura y orientación.

- La permeabilidad de los cerramientos se determina experimentalmente in situ mediante los ensayos de presurización.

- Las ecuaciones de equilibrio de presiones recogen todos los posibles factores despejando las incógnitas de la presión interior resultante y el caudal disponible.

- Finalmente, variando parámetros singulares de la ecuación, es posible cuantificar también la influencia de éstos en el valor total a considerar.

En conclusión, la aplicación de la metodología permite abordar la correcta caracterización de la disponibilidad de ventilación natural en las viviendas de los edificios.

Conocidos los resultados a la hora de implementar la exigencia de calidad del aire, quedan en evidencia las limitaciones del DB-HS3 a la hora de aplicarlo en una rehabilitación. En consecuencia, es conveniente plantear ampliar sus posibilidades: la solución propuesta sería proyectar un sistema individual de ventilación con caudal variable («Ventilación Cruzada Controlada») en cada vivienda. Sin embargo, previamente es preceptivo introducir en la normativa una serie de cambios:

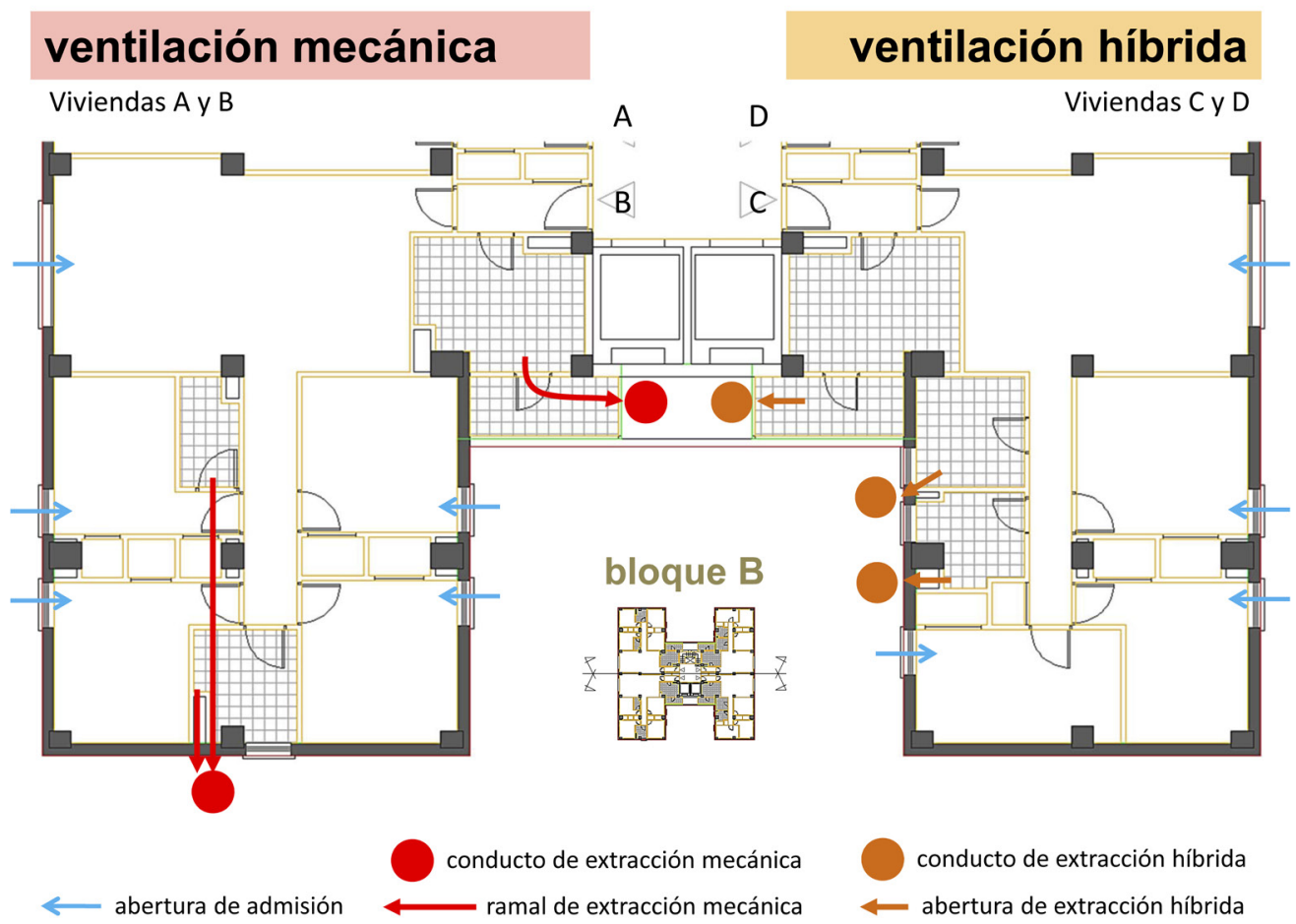

Figura 9. Propuesta de sistema de ventilación según DB HS-3. 
- Debería permitirse el sistema individual aunque sea un edificio plurifamiliar, lo que permitiría la actuación parcial en un número indeterminado de viviendas sin comprometer al resto del edificio. Este sistema facilita además el control personalizado de la ventilación en función de las necesidades de cada vivienda.

- Debería suprimirse las limitaciones en la ubicación de los aireadores y de las aberturas de expulsión, en favor de variantes cuyas combinaciones optimicen la difusión. Esa libertad podría facilitar la implementación de sistemas de recuperación de calor.

- Debería contemplarse que las aberturas de admisión trabajaran como mixtas en el sentido de permitir entre ellas una ventilación natural cruzada producto de las presiones y depresiones que se produzcan en sus fachadas, incorporando elementos de regulación del caudal en función de la velocidad del viento y la humedad.

- Cada vivienda debería tener su aspirador propio que extraiga el aire directamente de los locales húmedos, con una puesta en marcha supeditada a una determinada concentración de contaminante $\left(\mathrm{CO}_{2} \mathrm{o} \mathrm{HR}\right)$ o en el momento que se constate el uso de dichos locales, garantizando que estén siempre en depresión.

- Debería suprimirse la rígida limitación por la que las bocas de expulsión se ubican en cubierta, dado que en edificación abierta no se ven inconvenientes para que cada vivienda pueda contener su aspirador con la boca de expulsión en la propia fachada. En el caso de que el espacio al que se vierte el aire viciado fuera limitado (patio), cabrían dos posibilidades: que ese espacio sea exclusivamente de expulsión, o que se prevea también la admisión, en cuyo caso habría que prever favorecer la rápida dispersión del contaminante.

\section{AGRADECIMIENTOS}

Este trabajo forma parte del proyecto RepliCable and InnovaTive Future Efficient Districts and cities (CITyFiED), financiado por la Unión Europea a través del Seventh Framework Programme for research, technological development and demonstration activities (Grant Agreement number 609129).

\section{REFERENCIAS}

(1) Blázquez, T., Suárez, R., Sendra, J.J. (2015). Hacia una calibración de modelos energéticos: Caso de estudio del parque residencial español en clima mediterráneo. Informes de la Construcción, 67(540): e128, doi: http://dx.doi.org/10.3989/ ic.15.081.

(2) Suárez, R., Fragoso, J. (2016). Estrategias pasivas de optimización energética de la vivienda social en clima mediterráneo. Informes de la Construcción, 68(541): e136, doi: http://dx.doi.org/10.3989/ic.15.050.

(3) Sendra, J. J., Domínguez, S., León, A. L. (2011). Proyecto Efficacia: Optimización energética en la vivienda colectiva. Sevilla: Editorial Universidad Sevilla.

(4) ASHRAE. (2013). Ch.26.10. Natural Ventilation. Fundamental Handbook. Atlanta: ASHRAE.

(5) BSI. (1980). BS5925, Code of Practice for Design of Buildings: Ventilation principles and designing for natural ventilation. London: British Standard Institution.

(6) Chandra, S., Farinley, P.W., Houston, M. M. (1983). A handbook for designing ventilated building. Final Report FSECCR93-89. Cape Canaveral: Florida Solar Energy Centre.

(7) IDAE. (2010). Guía técnica de condiciones climáticas exteriores de proyecto. http://www.minetur.gob.es/energia/desarrollo/EficienciaEnergetica/RITE/Reconocidos/Reconocidos/CondicionesClimaticas.pdf.

(8) Instituto para la Diversificación y Ahorro de la Energía. (2015). Atlas eólico. http://atlaseolico.idae.es/meteosim.

(9) Blocken, B., Carmeliet, J., Stathopoulos, T. (2007). CFD evaluation of wind speed conditions in passages between parallel buildings: effect of wall-function roughness modifications for the atmospheric boundary layer flow. Journal of Wind Engineering and Industrial Aerodynamics, 95: 941-962.

(10) Kim, J. J., Baik, J. J. (2004). A numerical study of the effects of ambient wind direction on flow and dispersion in urban street canyons using the RNG k-e turbulence model. Atmospheric Environment, 38: 3039-3048, doi: http://dx.doi. org/10.1016/j.atmosenv.2004.02.047.

(11) Richards, P. J., Hoxey, R. P. (1993). Appropriate boundary conditions for computational wind engineering models using the k-e turbulence model. Journal of Wind Engineering and Industrial Aerodynamics, 46-47: 145-153, doi: http:// dx.doi.org/10.1016/0167-6105(93)90124-7.

(12) Sutton, O. G. (1949). Atmospheric turbulence. Quarterly Journal of the Royal Meteorological Society, $76: 108$.

(13) Moonen, P., Dorer, V., Carmeliet, J. (2011). Evaluation of the ventilation potential of courtyards and urban street canyons using RANS and LES, Journal of Wind Engineering and Industrial Aerodynamics, 99: 414-423, doi: http://dx.doi. org/10.1016/j.jweia.2010.12.012.

(14) Hertwig, D., Efthimiou, G. C., Bartzis, J. G., Leitl, B. (2012). CFD-RANS model validation of turbulent flow in a semiidealized urban canopy. Journal of Wind Engineering and Industrial Aerodynamics, 111: 61-72, doi: http://dx.doi. org/10.1016/j.jweia.2012.09.003.

(15) Sutton, O. G. (1936). The Logarithmic Law of wind Structure near the Ground. Quarterly Journal of the Royal Meteorological Society, 62: 124-127.

(16) Li, J., Ward, I. C. (2007). Developing Computational Fluid Dynamics conditions for urban natural ventilation study. En Proceedings: Building Simulation 2007 IBPSA. Beijing.

(17) Fluent Inc. (2003). FLUENT 6.1 User's Guide. Centerra Resource Park, Lebanon.

(18) Leitl, B. (2000). Validation data for microscale dispersion modelling. EUROTRAC Newsletter, 22: 28-32.

(19) Universität Hamburg. Compilation of Experimental Data for Validation of Microscale Dispersion Models. http://www. mi.uni-hamburg.de/CEDVAL_Validation_Data.427.0.html. 
(20) Padilla-Marcos, M. A., Feijó-Muñoz, J., Meiss, A. (2016). Wind velocity effects on the quality and efficiency of ventilation in the modelling of outdoor spaces. Building Services Engineering Research and Technology, 37: 33-50. doi: http:// dx.doi.org/10.1177/0143624415596441.

(21) Sherman, M. H. (1987). Estimation of Infiltration from Leakage and Climate Indicators. Energy and Buildings, 10: 81-86. 